\title{
A case of blood sweating: hematohidrosis syndrome
}

\author{
Roberto Maglie MD, Marzia Caproni MD
}

- Cite as: CMAJ 2017 October 23;189:E1314. doi: 10.1503/cmaj.161298

See related article at www.cmaj.ca/lookup/doi/10.1503/cmaj.170756

A

21-year-old woman was admitted to a general medical ward with a three-year history of selflimited episodes of bleeding from her palms and face despite no evidence of skin lesions. There was no obvious trigger for the bleeding, which could occur while she was asleep and during times of physical activity. She stated that more intense bleeding occurred during times of perceived emotional stress. Episodes lasted from one to five minutes.

Our patient had become socially isolated owing to embarrassment over the bleeding and she reported symptoms consistent with major depressive disorder and panic disorder. There was no history of psychosis.

Our differential diagnosis included factitious disorder and an uncommon condition known as hematohidrosis. Laboratory investigations (Appendix 1, available at www.cmaj.ca/ lookup/suppl/doi:10.1503/cmaj.161298/-/DC1) including a complete blood count and coagulation studies (prothrombin time, activated partial thromboplastin time, fibrinogen and D-dimer) were normal (Appendix 1). We found no evidence to support a diagnosis of factitious disorder.

We treated her depression and anxiety disorder with paroxetine and clonazepam, but her bleeding continued. During admission, we observed the discharge of blood-stained fluid from her face (Figure $1 \mathrm{~A}$ and Figure 1B). Based on the presence of erythrocytes on microscopic examination (excluding disorders that induce "coloured sweat" secretion, such as chromhidrosis and pseudochromhidrosis), we diagnosed hematohidrosis. Histologic analysis of the skin in an area of bleeding was normal (Figure 1C).

Hematohidrosis is an uncommon disease characterized by spontaneous discharge of "blood sweat" through intact skin. ${ }^{1}$ Various causes have been proposed, including systemic diseases, such as vicarious menses and coagulopathies (albeit historically reported in malaria, scurvy and epilepsy), exertion and psychogenic disorders, ${ }^{1}$ in which bleeding might be the result of exacerbated sympathetic nervous system activation. ${ }^{2}$

In the literature, there is no single explanation of the source of bleeding in hematohidrosis. Despite the fluid's sweat-like appearance, the hypothesis that blood passes through eccrine ducts, induced by abnormal constrictions and expansions of periglandular vessels, has not yet been proven. ${ }^{3}$ Bleeding has also reportedly occurred through areas without sweat glands ${ }^{2}$ or through the follicles, ${ }^{3}$ and the presence of dermal defects leading to blood-filled spaces exuding via follicular openings or directly into the skin surface has also been proposed. ${ }^{3}$

We treated our patient with propranolol ( $20 \mathrm{mg} /$ day), based on its use in similar cases in the literature, ${ }^{3}$ and this led to a marked reduction, although not a complete remission of her bleeding.

\section{References}

1. Holoubek JE, Holoubek AB. Blood, sweat and fear. A classification of hematidrosis. J Med 1996;27:115-33.

2. Wang Z, Yu Z, Su J, et al. A case of hematidrosis successfully treated with propranolol. Am J Clin Dermatol 2010;11:440-3.

3. Manonukul J, Wisuthsarewong W, Chantorn R, et al. Hematidrosis: a pathologic process or stigmata. A case report with comprehensive histopathologic and immunoperoxidase studies. Am J Dermatopathol 2008;30:135-9.

\section{Competing interests: None declared.}

This article has been peer reviewed.

The authors have obtained patient consent.

Affiliation: Department of Medical and Surgical Critical Care, Section of Dermatology, University of Florence, Florence, Italy

Correspondence to: Roberto Maglie, maglieroberto78@gmail.com 